\title{
The Tag Duplication Problem in an Integrated WSN for RFID-Based Item-Level Inventory Monitoring
}

\author{
Woohyung Chun \\ Department of ECE \\ SUNY at Stony Brook \\ Stony Brook, New York \\ Email:wchun@ece.sunysb.edu
}

\author{
Eric Noel \\ AT\&T Labs Research \\ Middletown, NJ \\ Email: eric.noel@att.com
}

\author{
K. Wendy Tang \\ Department of ECE \\ SUNY at Stony Brook \\ Stony Brook, New York \\ Email: wtang@ece.sunysb.edu
}

\begin{abstract}
Radio Frequency Identification (RFID) technology has been adopted in various supply chain management applications because of its fast and low-cost ability to identify items uniquely. In this paper, we consider the integration of a Wireless Sensor Network (WSN) with RFID to provide item-level inventory monitoring in retail stores. First, we present a basic formulation for RFID-based item-level inventory monitoring applications. With the formulation, we illustrate that tag duplication problems can exist in the network layer due to tags' mobility and overlaps of RFID readers' interrogation zones. In order to prevent the tag duplication, we propose a tag-filtering scheme by utilizing multihop routing capability of an integrated WSN with RFID. Our proposed filtering scheme is implemented in the nesC programming language and is executed in the TOSSIM simulator to demonstrate its efficiency. Our preliminary results indicate that our filtering scheme improves network latency and tag check-out rate.
\end{abstract}

Index Terms-RFID systems, Wireless Sensor Network, Ad Hoc Network

\section{INTRODUCTION}

Item-level deployment of RFID technology for real-time inventory tracking is a vision for many retailers such as Wal-Mart, Mark and Spencer and Tesco [1]. These retailers foresee that real-time inventory tracking of item-level units can eliminate long queues and reduce check-out times [1]. In addition, WSN is considered to be integrated with RFID technology to provide real-time, item-level, inventory tracking with environmental monitoring such as temperature and humidity for perishable goods [2]. In this paper, we consider a WSN integrated with RFID technology for real-time item-level inventory monitoring.

For item-level inventory monitoring applications in retail stores, RFID readers should be densely deployed with overlapped interrogation zones to cover all RFID tags. Therefore, tags located in the overlapped interrogation zones can be identified multiple times. If an RFID tag had the intelligence to distinguish which RFID reader it belonged to, overlapped interrogation zones would not produce any duplicated tags. However, tags having such capability are too costly [3]. In this paper, we consider low-cost passive RFID tags for itemlevel inventory monitoring applications.

Due to the overlapped interrogation zones of RFID readers, identified tags can appear multiple times in an item level inventory monitoring system. Previous researches [4] [5] identified the tag duplication problem in the overlapped interrogation zones and provided solutions in the physical layer. They assigned different frequencies to adjacent RFID readers and synchronized RFID readers to eliminate duplicated tags in the overlapped interrogation zones between adjacent RFID readers. However, duplicated tags can still appear in nonoverlapped RFID readers' interrogation zones because items attached to tags can be moved across RFID readers' interrogation zones. Thus, RFID readers need to communicate with one another to identify and eliminate duplicated tags.

To solve the tag duplication problem, we propose a network layer solution that filters duplicated tags. Some researches [6] [7] already pointed out the need for filtering redundant data in RFID based inventory monitoring system. However, they did not describe any specific method nor evaluate the efficiency of filtering. In this paper, we introduce a tag check-out rate as a metric to measure the efficiency of our proposed filtering scheme. For communication between RFID readers, we used wireless micro sensor nodes manufactured by Crossbow Technologies, Inc., called Motes [8]. The Motes have sensors for detecting environmental changes and are equipped with a wireless multihop routing protocol for sensor nodes' communication. Thus, the Motes can be used in an integrated WSN for RFID based item-level inventory monitoring with environmental sensing such as temperature and humidity for perishable goods. However, we caution that the Motes' transmitters only support low-rate communication (i.e. $38.4 \mathrm{kbps}$ for Mica2 and 250kbps for Micaz). Thus, the filtering scheme implemented in the Motes can only be applied for RFID based retail stores with a small business transaction rate. If a high business transaction rate is expected, the sensor nodes' transmitters should be replaced by faster one such as WiFi. On the other hand, since our proposed filtering scheme is implemented in the network layer, it can be applied regardless of the sensor nodes' transmission capabilities.

This paper is organized as follows: In Section II, we formulate the tag duplication problem based on a basic model for RFID item-level inventory monitoring applications. Section III proposes a solution for tag duplication based on a potential application of an integrated WSN for RFID itemlevel inventory monitoring. Section IV measures the efficiency of our proposed filtering scheme and Section V summarizes 


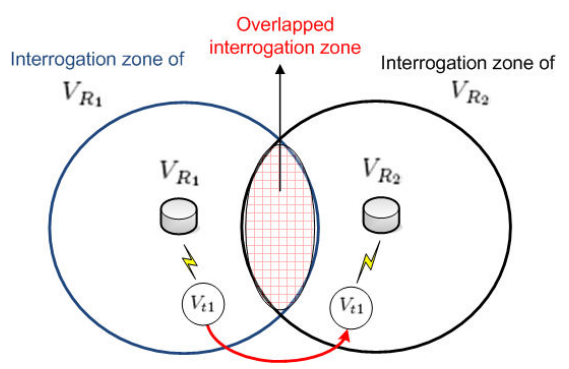

Fig. 1. Tag duplication by tags' mobility

our findings.

\section{BASIC FORMULATION}

RFID based item-level inventory monitoring system can be expressed as a graph $G=(V, E)$ where $V=V_{t} \bigcup V_{R}$ ( $V_{t}$ : RFID tags, $V_{R}$ : RFID readers) and $E=E_{t} \bigcup E_{R}$ $\left(E_{t}=\left(V_{t}, V_{R}\right)\right.$ : Channels for interrogating $V_{t}$ nodes, $E_{R}$ : Channels between $V_{R}$ nodes).

In $G$, tags are identified as

$$
\text { Ident : } V_{t} \times V_{R} \rightarrow V_{t}
$$

where Ident is the function that identifies tags. Therefore, $\operatorname{Ident}\left[\left(V_{t}, V_{R}\right)\right]$ is the set of identified tags in $\mathrm{G}$.

Since the RFID based item-level inventory monitoring application is required to track uniquely identified RFID tags, it must satisfy the following equation:

$$
\sum_{k=1}^{\left|V_{R}\right|}\left|\operatorname{Ident}\left[\left(V_{t}, V_{R_{k}}\right)\right]\right|=\left|V_{t}\right|
$$

where $\left|\operatorname{Ident}\left[\left(V_{t}, V_{R_{k}}\right)\right]\right|=$ number of identified $V_{t}$ nodes by $V_{R_{k}}$ nodes, $\left|V_{R}\right|=$ number of $V_{R}$ nodes in $\mathrm{G}$ and $\left|V_{t}\right|=$ number of $V_{t}$ nodes in G. That is, the number of all identified tags should be the same as the number of all deployed tags.

Since $V_{R}$ nodes should be densely deployed to cover all $V_{t}$ nodes in $G$, interrogation zones of $V_{R}$ nodes can be overlapped and $V_{t}$ nodes can be identified more than once. Therefore, the sum of identified tags in all interrogation zones, $\sum_{k=1}^{\left|V_{R}\right|}\left|\operatorname{Ident}\left[\left(V_{t}, V_{R_{k}}\right)\right]\right|$ can be larger than the actual number of tags, $\left|V_{t}\right|$. Furthermore, even though there is no overlapped interrogation zone, tag duplication can happen due to tags' mobility. For the case where $V_{t}$ nodes move around $G, V_{t}$ nodes can be identified more than once. Fig. 1 shows that $V_{t 1}$ is identified by both $V_{R_{1}}$ and $V_{R_{2}}$ when $V_{t 1}$ moves from non-overlapped interrogation zone of $V_{R_{1}}$ to non-overlapped interrogation zone of $V_{R_{2}}$.

In Fig. 2, we illustrate our basic model for RFID based itemlevel inventory monitoring. Interrogation zones of $V_{R}$ nodes are somewhat overlapped to cover all deployed $V_{t}$ nodes. The root node monitors $V_{t}$ nodes identified by $V_{R}$ nodes. All identified $V_{t}$ nodes are delivered via pre-assigned channels, $E_{R}$. For example, Ident $\left[\left(V_{t}, V_{R_{3}}\right)\right]$ can reach root node via $\left(V_{R_{3}}, V_{R_{1}}\right)$ and $\left(V_{R_{1}}, R o o t\right)$. When a WSN is integrated with RFID readers, $E_{R}$ can be mapped to routes between sensor nodes.
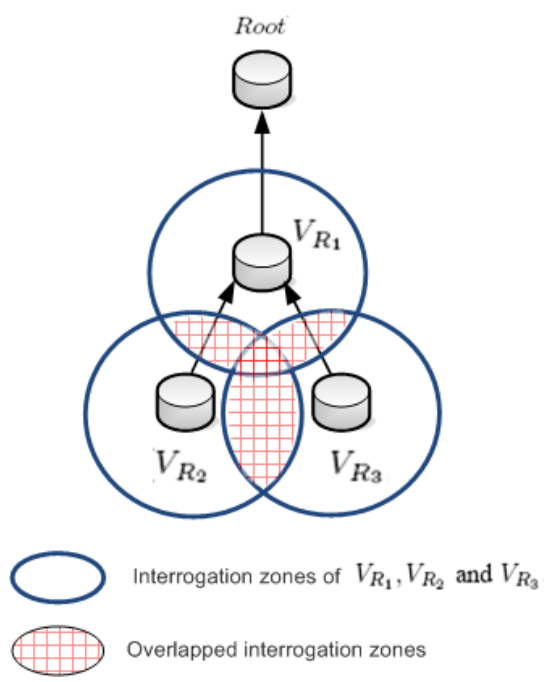

$V_{t}$ nodes are deployed in the interrogation zones of $V_{R_{1}}, V_{R_{2}}$ and $V_{R_{3}}$.

Fig. 2. Basic model for RFID based item-level inventory monitoring

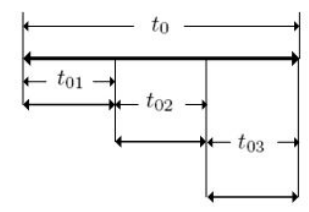

$t_{0}$ : a cycle for all $V_{R}$ nodes to interrrogate $V_{t}$ nodes $t_{01}$ : a time assigned to $V_{R_{1}}$ for interrogating $V_{t}$ nodes $t_{02}$ : a time assigned to $V_{R_{2}}$ for interrogating $V_{t}$ nodes $t_{03}$ : a time assigned to $V_{R_{3}}$ for interrogating $V_{t}$ nodes

Fig. 3. Time Division Interrogation is applied to Fig. 2

To show that tag duplication problems can happen in the network layer regardless of the interrogation method $E_{t}$, we consider two interrogation methods: Frequency Division Interrogation [4] and Time Division Interrogation [4].

Frequency Division Interrogation is the case where $\left|E_{t}\right|=$ $\left|V_{R}\right|$. Adjacent $V_{R}$ nodes use different frequencies (i.e. different $E_{t}$ ) to interrogate $V_{t}$ nodes. So, $V_{t}$ nodes can be identified multiple times through different frequency channels. For example, some $V_{t}$ nodes located in overlapped interrogation zones in Fig. 2 or moving between non-overlapped interrogation zones as shown in Fig. 1 can be identified by both $V_{R_{1}}$ and $V_{R_{2}}$ through $E_{t 1}=\left(V_{t}, V_{R_{1}}\right)$ and $E_{t 2}=\left(V_{t}, V_{R_{2}}\right)$.

Time Division Interrogation is the case where $\left|E_{t}\right|=1$. Adjacent $V_{R}$ nodes are synchronized to interrogate $V_{t}$ nodes in their overlapped interrogation zones at different times. Fig. 3 shows a synchronization of three $V_{R}$ nodes in Fig. 2. In Fig. $3, t_{0}$ is the cycle for interrogating all $V_{t}$ nodes at a time and is divided into $k$ time slots $\left(k=\left|V_{R}\right|\right)$. Each $V_{R}$ node only interrogates $V_{t}$ nodes in its assigned time slot. That is, $t_{01}$ is a time slot assigned to $V_{R_{1}}, t_{02}$ is assigned to $V_{R_{2}}$ and $t_{03}$ is assigned to $V_{R_{3}}$. However, when either $V_{t}$ nodes are located in the overlapped interrogation zones or $V_{t}$ nodes move between 


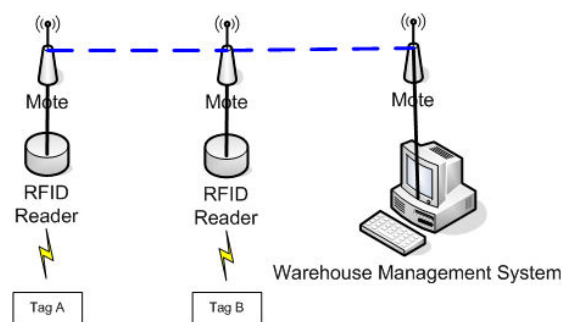

Fig. 4. Checking out tags through multihop routing

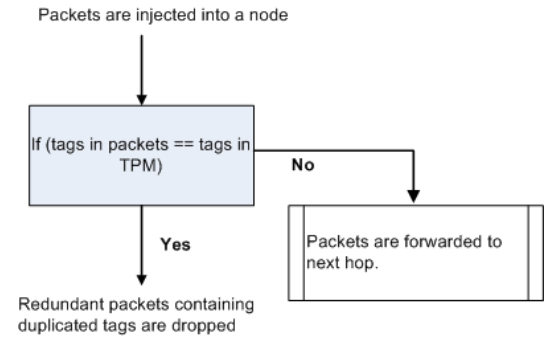

Fig. 5. TPM filtering scheme

time slots (i.e. $t_{01}, t_{02}$ and $\left.t_{03}\right), V_{t}$ nodes can be identified multiple times. For example, some $V_{t}$ nodes identified by $V_{R_{1}}$ in $t_{01}$ can also be identified by $V_{R_{2}}$ in $t_{02}$.

When either Frequency Division Interrogation or Time Division Interrogation is applied to the basic model in Fig. 2, $\sum_{k=1}^{3}\left|\operatorname{Ident}\left[\left(V_{t}, V_{R_{k}}\right)\right]\right|$ can be larger than $\left|V_{t}\right|$. That is, regardless of the interrogation method, there will be duplicated tags in RFID-based item-level inventory monitoring applications.

In Section III, we provide a solution for the tag duplication problem based on a potential application of a WSN integrated RFID for item-level inventory monitoring.

\section{PROPOSED SOLUTION FOR TAG DUPLICATION}

Our application network consists of wireless sensor nodes (Crossbow Technology Inc's Mote) integrated with RFID readers. The Mote integrated with an RFID reader supports multihop routing to forward packets to other Motes. The tag information identified by RFID readers in the wireless sensor nodes are sent back via the Motes' multihop routing to the warehouse management system (WMS) which is a real-time inventory tracking database as illustrated in Fig. 4.

To reduce the amount of duplicated traffic by overlapped interrogation zones and tagged items' mobility as illustrated in Section II, we propose that each intermediate node forwarding traffic to WMS filters out duplicated information. The filtering mechanism is achieved by recording and comparing the sent and forwarded tags' information in the local cache, called the temporary path marker, TPM [9]. A TPM is a table that records payloads of packets carrying tags' information. The TPM is updated whenever packets containing new tags' information are sent or forwarded in a sensor node. Fig. 5 shows the proposed filtering scheme with a TPM.

Basically, the size of a TPM depends on the number of tagged items in the interrogation zones of the RFID readers.

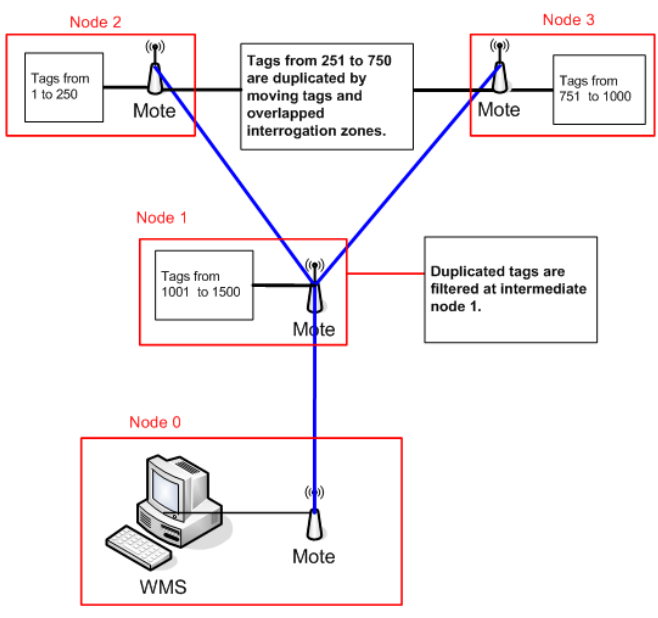

Fig. 6. Test application

Since commercial RFID readers for inventory monitoring have a large amount of memory rather than Motes' (64Mbytes for RFID readers, 128Kbytes for Motes ) [10], the TPM of each node can be located in the memory of its RFID reader. If one intermediate node receives the same (duplicated) tags' information with previously forwarded tags' information, the node does not forward the tags' information and just drops them according to the TPM.

\section{IMPLEMENTATION AND EFFICIENCY MEASUREMENT}

To measure the efficiency of our proposed filtering scheme described in Section III, we implemented our test application in the nesC programming language [11] and executed it in the TOSSIM [12] simulator. Our test application consists of 3 combined nodes (RFID reader + Mote) and 1 WMS node. Since TOSSIM has no model for RFID readers and tags, we simplified the identification of tags by generating a small amount of traffic containing tags in TOSSIM simulator. Each combined node has the list of identified tags in its interrogation zone. Tags used for our test application are 96-bit long as defined by the EPCglobal [13] standard. Since the payload size of TinyOS is 29bytes, two 96-bit tags' information can be encapsulated in one TinyOS message.

Fig. 6 shows our test application. Since node 2 and node 3 have 500 duplicated tags, node 2 can identify tags from 1 to 750 and node 3 can identify tags from 251 to 1000 . Therefore, tags from 251 to 750 are identified by both RFID Readers of node 2 and node 3. Table 1 shows parameters' mapping between our basic model in Fig. 2 and our test application in Fig. 6.

An item is checked out from the database of the WMS when its tag information is received by the WMS. The WMS has the list of all 1,500 tags at the beginning of the test application. As soon as items are checked out, they are removed from the list of WMS. When all tags are removed from the tag list of WMS, all products are sold out and our test application is completed. To model the purchasing rate within a node, we use the timer interrupt interval of a Mote. Each Mote in 
TABLE I

PARAMETERS' MAPPING BETWEEN BASIC MODEL AND TEST APPLICATION

\begin{tabular}{|c|c|c|}
\hline & Basic model in Fig. 2 & Test application in Fig. 6 \\
\hline & Root & Node 0 \\
\hline & $V_{R_{1}}$ & Node 1 \\
\hline & $V_{R_{2}}$ & Node 2 \\
\hline & $V_{R_{3}}$ & Node 3 \\
\hline & $\left|V_{t}\right|$ & 1500 \\
\hline & $\mid \operatorname{Ident}\left[\left(V_{t}, V_{R_{1}}\right)\right]$ & 500 \\
\hline & $\operatorname{Ident}\left[\left(V_{t}, V_{R_{2}}\right)\right]$ & 750 \\
\hline & $\operatorname{Ident}\left[\left(V_{t}, V_{R_{3}}\right)\right]$ & 750 \\
\hline $\mid \operatorname{Ident}\left[\left(V_{t}, V_{R_{1}}\right)\right.$ & ]$\cap \operatorname{Ident}\left[\left(V_{t}, V_{R_{2}}\right)\right]$ & 0 \\
\hline $\mid \operatorname{Ident}\left[\left(V_{t}, V_{R_{2}}\right)\right.$ & $\cap \operatorname{Ident}\left[\left(V_{t}, V_{R_{3}}\right)\right]$ & 500 \\
\hline$I \operatorname{dent}\left[\left(V_{t}, V_{R}\right.\right.$ & $\left\lceil\operatorname{Ident}\left[\left(V_{t}, V_{R_{3}}\right)\right]\right.$ & 0 \\
\hline
\end{tabular}

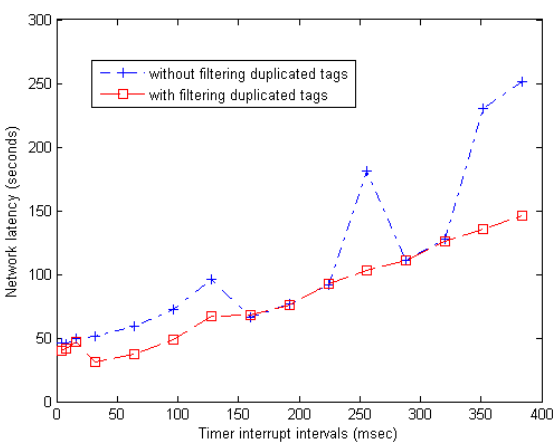

Fig. 7. Network latency

a node of our test application sends a packet whenever the timer interrupt is fired. Fig. 7 shows our simulation result for network latency. When our proposed filtering scheme is used, network latency is improved by $13 \%$.

We introduce the tag check-out rate as a metric to measure the efficiency of a retail store that integrates RFID and WSN for item-level inventory monitoring.

$$
\begin{aligned}
& \text { Tag check-out rate }= \\
& \frac{\text { total number of tags }}{\text { total time to check out all tags }}
\end{aligned}
$$

The tag check-out rate can be used as a design guideline in an integrated RFID with WSN infrastructure for retail stores.

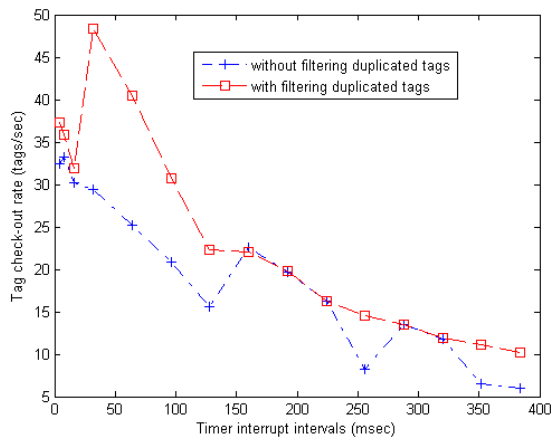

Fig. 8. Tag check-out rate
For instance, if the average customer generally purchases 30 items and the goal is to complete the customer's check out process in one second, the tag check-out rate should be equal to or larger than 30 tags/sec in Fig. 8.

\section{CONClusion}

In this paper, we discussed the tag duplication problem in an integrated WSN with RFID technology for real-time, item-level inventory monitoring. First, we described a basic formulation of RFID based item-level inventory monitoring. From the formulation, the tag duplication problem was identified in the network layer. We also explained that tags can be duplicated due to tags' mobility even if there is no overlapped interrogation. To eliminate tag duplication by both interrogation zones' overlaps and moving tags, we proposed a filtering scheme in the network layer. Our proposed filtering scheme was implemented in the nesC programming language and executed in the TOSSIM simulator to demonstrate its efficiency. Our results indicate that our filtering scheme improves network latency and tag check-out rate.

\section{ACKNOWLEDGMENT}

This research was partially supported by the National Science Foundation under Grant No. EEC-0332605. Any opinions, findings and conclusions or recommendations expressed in this article are those of the authors and do not necessarily reflect the views of the National Science Foundation.

\section{REFERENCES}

[1] George Roussos,"Enabling RFID in Retail," Computer, IEEE, Volume 39, Issue 3, March 2006 Pages 25-30.

[2] Roy Want,"An introduction to RFID technology," Pervasive Computing, IEEE, Volume 5, Issue 1, Jan-March 2006 Pages 25-33.

[3] RFID Journal, "RFID System Components and Costs," RFID Journal Web, http://www.rfidjournal.com/article/articleview/1336/1/129/

[4] Engels, D.W., Sarma, S.E.,"The Reader Collision Problem, "Systems, Man and Cybernetics, 2002 IEEE International Conference on Volume 3, 6-9 Oct. 2002 Page(s):6 pp. vol.3

[5] Carbunar, B., Ramanathan, M.K., Koyuturk, M., Hoffmann, C. Grama, A., "Redundant reader elimination in RFID systems," Sensor and Ad Hoc Communications and Networks, 2005. IEEE SECON 2005. 2005 Second Annual IEEE Communications Society Conference on 26-29 Sept., 2005 Page(s):176 - 184.

[6] Niederman, F., Mathieu, R. G., Morley, R., and Kwon, "Examining RFID applications in supply chain management," Communications of the ACM, Volume 50, Issue 7, July 2007 Pages 92-101.

[7] IBM, "Item-level RFID technology redefines retail operations with realtime, collaborative capabilities.", http://www.ibm.com

[8] Crossbow Web, http://www.xbow.com

[9] Woohyung Chun, Wendy Tang, "Multicasting in Wireless Sensor Networks," The 1st International Conference on Next Generation Network(NGNCON 2006)., Jeju, Korea, July 9-13, 2006 Page(s):251 - 253.

[10] Motorola Web, http://www.motorola.com/rfid

[11] Gay, D., Levis, P., von Behren, R., Welsh, M., Brewer, E., and Culler, D., "The nesC language: A holistic approach to networked embedded systems," In Proceedings of the ACM SIGPLAN 2003 Conference on Programming Language Design and Implementation, San Diego, California, USA, June 09 - 11, 2003, PLDI '03. ACM Press, New York, NY, 1-11.

[12] Levis, P., Lee, N., Welsh, M., and Culler, D., "TOSSIM: accurate and scalable simulation of entire tinyOS applications," In Proceedings of the 1st international Conference on Embedded Networked Sensor Systems, Los Angeles, California, USA, November 05 - 07, 2003, SenSys '03. ACM Press, New York, NY, 126-137.

[13] EPCGlobal Web, http://www.epcglobalinc.org 